\title{
Thermoelectric power in the quantum-Hall regime at very low temperatures
}

\author{
B. Tieke ${ }^{\mathrm{a}, *}$, R. Fletcher ${ }^{\mathrm{b}}$, S.A.J. Wiegers ${ }^{\mathrm{a}}$, U. Zeitler ${ }^{\mathrm{a}}$, J.C. Maan ${ }^{\mathrm{a}}$, C.T. Foxon ${ }^{\mathrm{c}}$, J.J. Harris ${ }^{\mathrm{d}}$ \\ a High Field Magnet Laboratory, University of Nijmegen, 6525 ED Nijmegen, The Netherlands \\ 'Physics Department, Queen's University, Kingston, Ontario, Canada \\ 'Department of Physics, University of Nottingham, Nottingham NG7 2RD, UK \\ ${ }^{\mathrm{d}}$ Electronic and Electrical Engineering, University College, London WCIE 7JE, UK
}

\begin{abstract}
We have measured the thermoelectric power (TEP) of high mobility 2DEGs in the integer and fractional quantum Hall regime down to $100 \mathrm{mK}$. At low temperatures, fluctuation-like structures in the TEP are observed. Below $160 \mathrm{mK}$ the TEP diverges around the filling factor $v=\frac{1}{5}$ where the resistivity enters the insulating phase. The temperature dependence of the TEP at filling factors $v=\frac{1}{2}$ and $\frac{1}{4}$ resembles that of the zero field TEP enhanced by a constant factor. This is interpreted in terms of the phonon drag of composite fermions.
\end{abstract}

\section{Introduction}

The galvanomagnetic properties of two-dimensional electron gases (2DEGs) display a number of interesting phenomena such as the integer quantum-Hall-effect (IQHE) [1], the fractional quantum-Hall-effect (FQHE) [2], and the appearance of an insulating phase (IP) at low filling factors which has been attributed to a pinned Wigner solid [3].

Because the thermoelectric power (TEP) of 2DEGs in $\mathrm{GaAs}-\mathrm{Ga}_{1-} \mathrm{Al}_{x} \mathrm{As}$ heterostructures is usually dominated by phonon drag, it provides a direct measure of the electron-phonon interaction $[4,5]$ and can therefore yield complementary information on the nature of the 2DEG compared to resistivity (which is determined by electron-impurity scattering). The recent review by

* Corresponding author.
Gallagher and Butcher [6] on the TEP of 2DEGs provides a good introduction to the present work. The relation between the applied temperature gradient (which supplies the driving force) $\nabla T$ and the induced electric field $\boldsymbol{E}$ defines the TEP tensor $S: E=S \nabla T$. With a magnetic field $B$ applied perpendicular to the 2DEG there are only two independent components of $\boldsymbol{S}$, the thermopower $S_{x x}$ and the Nernst-Ettingshausen coefficient $S_{y x}$.

In this paper we will report the first experimental results concerning the $S$ of a 2DEG at temperatures $T$ down to $100 \mathrm{mK}$ in high magnetic fields. We will show that with decreasing temperature additional structures in the TEP appear and, at the lowest temperatures, a diverging TEP is observed at filling factors where the IP in resistivity measurements shows up. Furthermore, we will present the temperature dependence of the TEP at $v=\frac{1}{2}$ and $v=\frac{1}{4}$ and compare it to the zero field TEP in the framework of the composite fermion model. We will mainly concentrate on $S_{x x}$ but most statements also apply to $S_{y x}$. 


\section{Experimental}

We investigated three high mobility $\left(\mu \simeq 200 \mathrm{~m}^{2} / \mathrm{Vs}\right.$ ) GaAs- $-\mathrm{Ga}_{1-x} \mathrm{Al}_{x} \mathrm{As}$ heterostructures (kindly supplied by Philips Research Laboratory, Redhill, England) labeled G645 $\left(n=0.75 \times 10^{11} \mathrm{~cm}^{-2}\right)$, G647 $\left(n=0.43 \times 10^{11} \mathrm{~cm}^{-2}\right)$, and $\mathrm{G} 650\left(n=1.0 \times 10^{11} \mathrm{~cm}^{-2}\right)$. Each 2DEG was grown on a semi-insulating GaAs substrate and was formed into a Hall bar with eight AuGeNi diffused ohmic contacts.

The experiments were carried out in the vacuum space of a ${ }^{3} \mathrm{He}-{ }^{4} \mathrm{He}$ dilution refrigerator adapted for use in high magnetic fields by having no metallic heat exchangers [7]. To avoid further eddy current heating, the sample holder was made of plastic and the only metal parts were in the thermal anchoring of the sample to the mixing chamber. The free-standing sample was In-soldered to the cold finger and the thermal gradient was produced by a heater glued to the free end. Typical temperature differences were a few tens of $\mathrm{mK}$ across the 2DEG and never exceeded $10 \%$ of the absolute temperature. Both $\nabla T$ and $T$ were measured with two $\mathrm{RuO}_{2}$ film resistors mounted on the rear side of the substrate. Special care was taken to eliminate all extraneous sources of heat loss from the sample.

As a check for the thermometry we have measured the thermal conductivity $\lambda$ of the samples. $\lambda$ is determined by the phonons in the three-dimensional substrate. Experimentally, $\lambda$ follows the $T^{3}$-dependence expected for a constant phonon mean free path which is limited by boundary scattering.

The TEP was measured using pulsed heating and detected at twice the frequency of the electrical heater current using a lock-in technique. The measuring frequencies were in the range $2-12 \mathrm{~Hz}$. We verified the thermal response time being fast enough by obtaining the same results (within $10 \%$ ) for different frequencies.

\section{Results and discussion}

Although at $T>400 \mathrm{mK}$ the TEP is very much smaller in the IQH regime than in the FQH regime, ${ }^{1}$ the IQH TEP becomes comparable to, and even bigger than, the FQH TEP for $T<350 \mathrm{mK}$ (see Fig. 1). At the lowest temperatures, the most pronounced structure is the peak between filling factors $v=1$ and $v=2$, i.e. between the two lowest spin-split Landau levels.

With decreasing $T$ the TEP increasingly exhibits fluctuation-like structure. This is very reproducible for

\footnotetext{
${ }^{1}$ For a detailed description of the TEP in this temperature range, see Ref. [5].
}

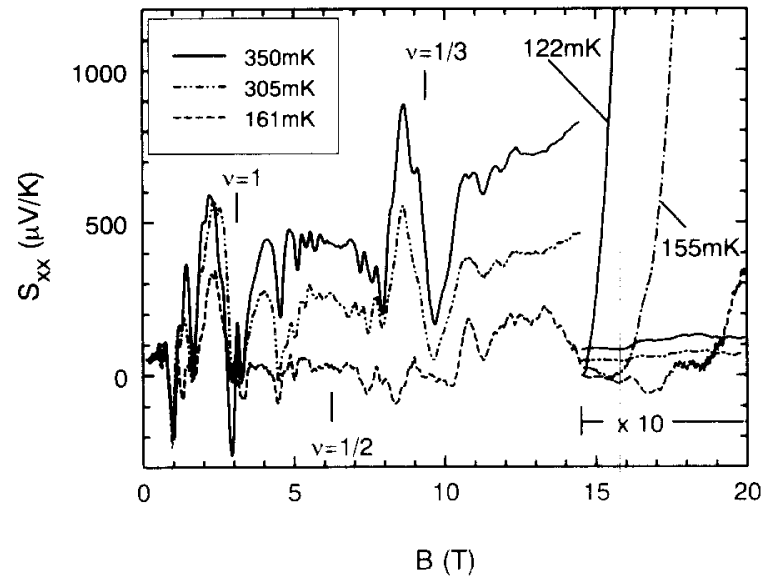

Fig. 1. The low-T thermopower $S_{x x}$ of G645. With decreasing $T$ reproducible fluctuation-like structures appear. In the field range of the insulating phase in the resistivity the TEP is diverging. For clarity the low-B parts of $S_{x x}$ below $160 \mathrm{mK}$ are omitted.

different field sweeps and is similar at different $T$, but the details of the structure are different when $\boldsymbol{B}$ is reversed. This means that the measured $S_{x x}$ and $S_{y x}$ have contributions which are both even and odd in $\boldsymbol{B} . S_{x x}$ is expected to be even under reversal of $B$ and $S_{y x}$ should be odd, but experimentally there is usually some admixture of the one into the other because of imperfect contact alignment. However, in the present experiments such a geometric effect can be excluded as being responsible for the observed behaviour because the unexpected contributions are essentially only seen in the IQH regime and not in the FQH regime, e.g. the odd part of $S_{x x}$ vanishes for $v<1$ (with some deviation around $v=\frac{1}{3}$ ) even though the amplitude of $S_{y x}$ in the FQH range is rather large.

Another interesting feature is that we find a similar behaviour for the $T$ dependence of $S_{x x}$ at filling factors of $\frac{1}{2}$ and $\frac{1}{4}$ as we do with the zero field TEP (see Fig. 2). At $v=\frac{1}{2}\left(\frac{1}{4}\right), S_{x x}$ is about a factor of $20(50)$ larger than at zero $B$. We interpret the measured TEP at $v=\frac{1}{2}$ and $\frac{1}{4}$ as the "zero field phonon drag TEP" of the composite Fermions (CF) at these points [8] and deduce that the CFs have a stronger interaction with the phonons than noninteracting electrons. CFs are quasi-particles consisting of an electron bound to an even number of flux quanta. The effective magnetic field for CFs is zero at filling factors with even denominator $\left(v=\frac{1}{2}, v=\frac{1}{4}\right.$, etc.) [9].

At zero B, the phonon drag TEP $S^{g}$, which is usually the main contribution to the TEP $[4,5]$ can be expressed $[10,6]$ as

$S^{\mathrm{g}}=-\frac{v L m^{*}}{e T \tau_{\mathrm{ep}}}=-\rho^{\mathrm{ph}} \frac{n e v L}{T}$, 


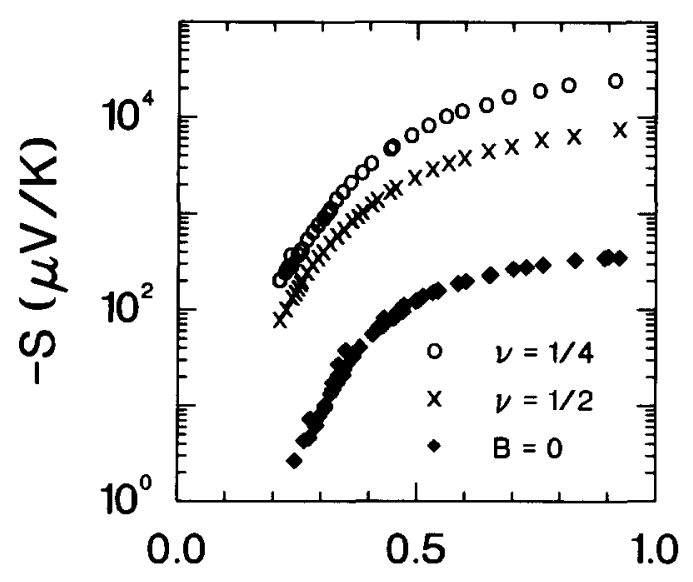

Temperature (K)

Fig. 2. The temperature dependence of $S_{x x}$ at filling factors $v=\frac{1}{2}$ and $v=\frac{1}{4}$ compared the zero field TEP of G647.

where $v$ is the sound velocity in the GaAs substrate, $L$ the phonon mean free path, $m^{*}$ the effective mass of the electrons, $\tau_{\text {ep }}$ the scattering time between the three-dimensional phonons and the $2 \mathrm{DEG}$, and $\rho^{\mathrm{ph}}=m^{*} / n e^{2} \tau_{\mathrm{ep}}$ is the resistivity due to electron-phonon scattering. A similar relation still holds with $B$ present [11]. Eq. (1) can be applied to CFs in which case $m^{*} \tau_{\mathrm{ep}}^{-1}$ is replaced by $m_{\mathrm{cf}}^{*} \tau_{\mathrm{cfp}}^{-1}$, with $m_{\mathrm{cf}}^{*}$ being the effective mass of the CFs and $\tau_{\text {crp }}^{-1}$ the CF-phonon scattering rate [12]. Leadley et al. [13] report a slightly $B$-dependent effective mass $m_{\mathrm{cf}}^{*} \approx 0.5 m_{\mathrm{e}}$ for the $v=\frac{1}{2} \mathrm{CF}$, where $m_{\mathrm{e}}$ is the free electron mass, and a similar value at $v=\frac{1}{4}$ but with a stronger $B$ dependence. According to Eq. (1), an increase of $m^{*}$ from 0.067 to $0.5 \mathrm{~m}_{\mathrm{e}}$ results in an increase of $S_{x x}$ by about a factor of 8 . The experimentally found enhancement of $S_{x x}$ by $\sim 20$ and $\sim 50$, respectively, would imply a phonon scattering rate which is about 2-3 times stronger for the $v=\frac{1}{2} \mathrm{CF}$ than for non-interacting electrons and, with the same $m_{\mathrm{cf}}^{*}$, another factor of 2-3 stronger for the $v=\frac{1}{4} \mathrm{CF}$.

At $T<160 \mathrm{mK}$ a diverging TEP is observed (see Fig. 1) in the field range where the IP develops in resistivity measurements (around $v=\frac{1}{5}$ ). At $T>200 \mathrm{mK}$ the TEP decreases strongly with $T$ in this field range. A diverging $S_{x x}$ has been reported in Ref. [14] in a $2 \mathrm{DHG}$ for filling factors around $v=\frac{1}{3}$. Essentially the same behaviour is shown by $S_{y x}$ but we note that the diverging part does not change sign when $B$ is reversed, whereas the rest of the structure in the $F Q H$ regime does.

\section{Conclusions}

We have measured the TEP of 2DEGs in $B \leq 20 \mathrm{~T}$ and down to $100 \mathrm{mK}$. At low $T$ we observe field-dependent fluctuations, mainly in the IQH regime, and a diverging TEP around $v=\frac{1}{5}$. The $T$ dependence at $v=\frac{1}{2}$ and $v=\frac{1}{4}$ is found to be similar to the zero field TEP and is interpreted using the composite fermion model. Further experimental as well as theoretical investigations concerning these observations are in progress.

\section{Acknowledgements}

We would like to acknowledge the stimulating discussions with V.I. Fal'ko, especially on the topic of composite fermions.

\section{References}

[1] K. von Klitzing, G. Dorda and M. Pepper, Phys. Rev. Lett. 45 (1980) 494.

[2] D.C. Tsui, H.L. Störmer and A.C. Gossard, Phys. Rev. Lett. 48 (1982) 1559.

[3] H.W. Jiang, H.L. Störmer, D.C. Tsui, L.N. Pfeiffer and K.W. West, Phys. Rev. B 44 (1991) 8107 and references therein.

[4] R. Fletcher, J.C. Maan, K. Ploog and G. Weimann, Phys. Rev. B 33 (1986) 7122.

[5] U. Zeitler, J.C. Maan, P. Wyder, R. Fletcher, C.T. Foxon and J.J. Harris, Phys. Rev. B 47 (1993) 16008; U. Zeitler, R. Fletcher, J.C. Maan, C.T. Foxon, J.J. Harris and P. Wyder, Surf. Sci. 305 (1994) 91.

[6] B.L. Gallagher and P.N. Butcher, in: Handbook on Semiconductors, Vol. 1, eds. T.S. Moss and P.T. Landsberg (Elsevier, Amsterdam, 1992) p. 721.

[7] J.A.A.J. Perenboom, K. van Hulst, S.A.J. Wiegers and J.C. Maan, Physica B 201 (1994) 507.

[8] J.K. Jain, Adv. Phys. 41 (1992) 105.

[9] B.I. Halperin, P.A. Lee and N. Reed, Phys. Rev. B 47 (1993) 7312.

[10] C. Herring, Phys. Rev. 96 (1954) 1163.

[11] V.I. Fal'ko and S.V. Iordanskii, J. Phys.: Condens. Matter 4 (1992) 9201.

[12] U. Zeitler, B. Tieke, S.A.J. Wiegers, J.C. Maan, R. Fletcher, V.I. Fal'ko, C.T. Foxon and J.J. Harris, in: Proc. 11th Internat. Conf. on High Magnetic Fields in Semiconductor Physics (SEMIMAG-94), Cambridge, MA, 1994, to be published.

[13] D.R. Leadley, R.J. Nicholas, C.T. Foxon and J.J. Harris, Phys. Rev. Lett. 72 (1994) 1906.

[14] V. Bayot, X. Ying, M.B. Santos and M. Shayegan, Europhys. Lett. 25 (1994) 613. 